\title{
Experiencias en la selección de sensores para el sistema de determinación de orientación de satélites pequeños tipo CubeSat
}

\author{
Experiences in the sensors selection for the attitude \\ determination system of small satellites type CubeSat
}

\author{
David Arévalo ${ }^{+}$y Ronald Hurtado-Velasco*(iD \\ Escuela de Ciencias Exactas e Ingeniaría \\ Universidad Sergio Arboleda \\ Calle 74 No 14-14, Bogotá, Colombia
}

\begin{abstract}
Resumen. Un sistema de orientación activo para satélites es prioritario cuando se desea apuntar algún instrumento, como una cámara para obtener imágenes de la superficie terrestre. Los métodos que guían la implementación de los sensores para sistemas de orientación están formulados para programas y proyectos espaciales en los cuales los satélites son de gran tamaño, con amplios recursos económicos y humanos, esos métodos no se pueden implementar en su totalidad en un proyecto CubeSat con recursos limitados. Nuestra propuesta empieza con una definición y análisis de requerimientos. Se seleccionaron algunos sensores para la realización de experimentos y toma de medidas para el diseño y desarrollo de una tarjeta de circuito impreso que permita la integración de los sensores del sistema de orientación y se comunique con el computador del satélite. Presentamos nuestra experiencia en la evaluación de los sensores y consideraciones de diseño para su implementación en un sistema de control y determinación de orientación para satélites pequeños. Además de la selección de los componentes, presentamos las fases de requerimientos, selección de componentes y consideraciones de diseño para el uso de los sensores del sistema de orientación del satélite. Consideramos que nuestra experiencia es útil para grupos de trabajo con poca experiencia o que quieren incursionar en una misión satelital pequeña con el desarrollo de su sistema de orientación.
\end{abstract}

Palabras Claves. CubeSat; Sistema de Determinación de Orientación; Libertad 2.

\begin{abstract}
An active determination system for satellites has priority when it is wanted to point some instrument, such as a camera, in order to obtain images of the Earth's surface. The methods that guide the implementation of sensors for determination systems are formulated for space programs and projects in which satellites are large, with extensive economic and humans resources, these methods cannot be fully implemented in a CubeSat project with limited resources. Our proposal begins with the definition and analysis of requirements. Some sensors were selected for conducting experiments and take measurements in order to design and development a print circuit board that would allow the integration of the sensors of the orientation system and communicates with the satellite computer. We present our experience in the evaluation of sensors and design considerations for its implementation in an Attitude Determination and Control System for small satellites. In addition to the selection of the components, we present the phases of requirements, selection of components and design considerations for using sensors of the satellite attitude system. We believe that our experience
\end{abstract}




\begin{abstract}
is useful for working groups with few experience or who want to move into a small satellite mission with the development of its attitude system

Keywords. CubeSat; Attitude Determination System; Libertad 2.

Como Citar. D. Arévalo y R. Hurtado-Velasco, "Experiencias en la selección de sensores para el sistemas de determinación de orientación de satélites pequeños tipo CubeSat", Jou. Cie. Ing., vol. 12, no. 1, pp. 166-176, 2020. doi:10.46571/JCI.2020.1.15
\end{abstract}

Recibido: 15/09/2019 Revisado: 08/05/2020 Aceptado: 11/07/2020

\title{
1. Introducción
}

El desarrollo del estándar CubeSat por la Universidad de Stanford y el Politécnico de California en 1999 [1, 2, 3, 4], abrió la puerta a universidades e industrias para realizar misiones satelitales de corto plazo y a bajo costo de operación y producción, con objetivos de investigación, tecnológicos y comerciales $[5,6,7]$.

Un CubeSat es un satélite en forma de cubo de $10 \mathrm{~cm}$ de $1 \mathrm{~kg}$, también conocido como un CubeSat 1U, el cual puede ser combinado para formar uno mas grande, por ejemplo, si se combinan tres de estos en linea se tendrá un CubeSat $3 \mathrm{U}\left(10 \times 10 \times 30 \mathrm{~cm}^{3}\right)$.

Para algunas universidades, los CubeSat se han consolidado como una excelente plataforma para involucrar a estudiantes y profesores en proyectos de desarrollo tecnológico y científico y así apropiar nuevos conocimientos. Es el caso del AOXiang un Cubesat 12U [8], desarrollado por Universidad Politécnica de Northwestern (China), cuyos objetivos fueron desarrollar el sistema de despliegue para 12U, investigar la viabilidad de utilizar luz solar polarizada para la determinación de orientación del satélite, y realizar investigaciones de microgravedad utilizando un gravímetro miniaturizado, probar una computadora a bordo con tolerancia a fallas utilizando la tecnología System On the Programmable Chip (SOPC), y obtener experiencia en el desarrollo del CubeSat y sus subsistemas. En este proyecto participaron mas de 50 estudiantes.

En el caso de industrias podemos citar a NASA Goddard Space Flight Center, quienes desarrollaron el Firefly un CubeSat 3U, para explorar la relación entre los rayos y los destellos de Rayos Gamma Terrestre (TGF) mediante el uso de Rayos Gamma [9].

La Universidad Sergio Arboleda esta desarrollando la misión satelital Libertad 2, un Cubesat 3U, para la observación de la Tierra desde órbitas bajas [10]. Para cumplir con este objetivo el satélite debe contar con un sistema de orientación activo para apuntar la cámara, este sistema es denominado ADCS (Attitude Determination and Control System). El ADCS precisa de un conjunto de sensores para determinar su orientación y luego apuntar la cámara activando uno o varios de sus actuadores $[11,12]$.

En el desarrollo de nuestra primera misión satelital, Libertad 1 [13], no se contó con un sistema activo de control; se uso un imán permanente para que actuara con el campo magnético terrestre. Para el Libertad 2 [10], se requiere un sistema de control activo, sistema en el cual el nuevo equipo no tenía experiencia. El equipo se encargó de estudiar el sistema de orientación lo que ayudó a entender su funcionamiento y aplicación, pero se encontró poca información que guiara en el diseño a personas sin experiencia. En cuanto a los métodos que guían la implementación de los sensores para los sistemas de orientación, estos están enfocados a programas y proyectos espaciales en los cuales los satélites son de gran tamaño, con amplios recursos económicos y humanos, esas metodologías no se pueden implementar en su totalidad en un proyecto CubeSat con recursos limitados.

Los sistemas de orientación como los usados en los satélites son actualmente usados en múltiples aplicaciones que involucran movimiento, tales como el estudio de la rotación de cuerpos, dispositivos de juegos y realidad virtual, orientación de pantallas, robótica, aeronaves, etc [14]. Aunque existen diferentes empresas que comercializan sistemas de determinación de orientación que pueden ser usados para múltiples aplicaciones, no todas pueden usarse en 
aplicaciones satelitales y tampoco se ajustan a todas las necesidades de la misión que estamos desarrollando. En la documentación revisada de misiones satelitales similares a la nuestra: CubeSats ESTCube-1 [15], CubETH [16], ANTELSAT [17], UYS-1 [18], RAX [19] y UWE-3 [20], los equipos centran sus reportes en la forma en que diseñaron e implementaron el sistema de orientación de cada misión, pero no presentan una guía metodológica tanto para la selección de los sensores como para el diseño del sistema de orientación.

El método que usamos se complementa con el que se ha presentado en "Guía Metodológica para el Diseño de Subsistemas de Potencia y Orientación en NanoSatélites" [21], enfocado en el Subsistema de Potencia y Energía (Electrical Power System, EPS) y el Subsistema de Orientación (Attitude Determination and Control Systema, ADCS).

Presentamos nuestra experiencia y el método que usamos para la selección de los sensores del sistema de orientación, proponemos las fases de requerimientos y la selección de componentes. Además, el diseño de una tarjeta electrónica para los sensores, la que se comunica con el computador principal y cumple con los protocolos del bus de comunicaciones de nuestro satélite. Con este método que se puede ajustar a las necesidades y recursos limitados de un proyecto de formación académica, hemos logrado apropiación de conocimiento en el área de sistemas de orientación para satélites pequeños.

\section{Consideraciones para el desarrollo del método}

De todas las actividades que hemos desarrollado para conocer y entender el sistema de orientación de un satélite pequeño, y teniendo en cuenta el objetivo del Libertad 2, nos hemos enfocado en la definición de los requerimientos de algunos sensores (magnetómetro, sensor de luz), la selección de los sensores, las pruebas de funcionamiento y el diseño de los circuitos para el acondicionamiento de las señales. A partir de esas actividades proponemos un método orientado a la selección de los sensores para un sistema de orientación de una misión satelital tipo CubeSat.

\subsection{Definición de requerimientos de los sensores}

Los requerimientos de los sensores son función del sistema general de orientación y de los objetivos de la misión. Los requerimientos generales contemplan modos de operación, grados de precisión, consumo de energía, tamaño y tipo de actuadores. Para nuestro caso, estos requerimientos fueron presentados por F. Sierra et al. en [21]. Hemos definido el tipo y cantidad de sensores que se van a usar, lo cual lleva a estudiar la naturaleza física de la variable medida y establecer los requerimientos funcionales de cada sensor.

De la revisión de documentación de sistemas de orientación de diferentes misiones satelitales tipo CubeSat, se encontró que aunque es posible calcular la orientación con al menos una variable física mediante un algoritmo de estimación, cada misión usa al menos dos tipos de sensores con un respectivo algoritmo de estimación o determinación. La siguiente es una lista de sensores mas comunes usados en sistemas de orientación [22]:

- Giroscopio

- Magnetómetro

- Sensor de sol

- Sensor de estrellas

De estos, los más usados son el giroscopio y el magnetómetro, los cuales dependiendo de su calidad y combinación en un algoritmo logran obtener un buen nivel de precisión en el cálculo de la orientación. Agregar más medidas físicas puede ayudar a la precisión, pero entra en juego la relación costo-beneficio de la misión.

Se recomienda que el rango de medición del giroscopio sea igual o superior a $1000 \% \mathrm{~s}$ CUBETH [16]. Para el magnetómetro considere que el campo magnético terrestre varia de 24000 
- $66000 \mathrm{nT}$ (0.24 - $0.66 \mathrm{G})$ [23]. De acuerdo al rango que se quiere medir se puede recomendar o seleccionar una sensibilidad determinada como requerimiento del sensor.

\subsection{Selección de sensores}

Para la selección de los sensores hay que tener en cuenta su rango de medición, sensibilidad y el offset del mismo. Adicionalmente considerar la sensibilidad a la radiación, rango de temperatura, consumo de energía, tamaño, precio, tecnología, tipo de salida, etc.

Teniendo en cuenta que en este tipo de proyectos académicos el presupuesto es bajo, se necesita usar componentes tipo "Commercial Off the Shelf" (COTS), con el propósito de disminuir costos y agilizar el proceso de diseño. Para este tipo de componentes es importante que no sean sensibles a la radiación y que tengan un amplio rango de temperatura de operación. Aunque no es común en la mayoría de componentes electrónicos, ciertos materiales se ven afectados por el vacío del espacio lo cual genera un fenómeno llamado desgasificación (Outgassing), el cual puede afectar a otros componentes. Para la selección de materiales la NASA Goddard Space Flight Center cuenta con una lista de materiales de baja desgasificación [24].

Busque referencias de distintos fabricantes y compare sus características, de esa manera podrá seleccionar uno que se adapte a las necesidades o restricciones de su misión.

\subsection{Prueba de sensores}

Ante la ausencia de equipos adecuados para probar los sensores (sensibilidad, offset, etc.) es necesario confiar en las especificaciones del fabricante, dado que son los que tienen los recursos necesarios para probar sus tecnologías. Sin embargo si se requiere realizar algunas pruebas específicas del funcionamiento de algún sensor procure simular las condiciones de la variable física que mide el sensor.

Para el caso del giroscopio, una mesa rotatoria puede ayudar a verificar si las medidas cambian respecto al giro de la mesa a ciertas velocidades. Para el magnetómetro podría usarse una bobina de Helmholtz o como alternativa algún imán que no supere el rango de medición del sensor.

\subsection{Consideraciones de diseño}

De acuerdo a las necesidades y recursos de la misión, el uso de los sensores puede variar, ya sea por el tipo de salida de los sensores (análoga, digital), configuración mediante comunicaciones digitales (I2C, SPI, UART), voltaje alimentación o voltaje de salida de las señales. Esas condiciones de los sensores determinan el tipo de acondicionamiento de la señal, la interfaz con la unidad de procesamiento y circuito de alimentación.

Para el sensor de sol se pueden usar fotodiodos, uno en cada cara del satélite, de manera tal que cuando la incidencia de luz del sol sea directa se genere un máximo voltaje de salida y este decrezca en proporción al ángulo de incidencia de luz hasta un valor de cero cuando no haya luz. Otra alternativa es usar el voltaje de salida de los paneles solares que vayan en cada una de las caras del CubeSat.

\section{Resultados}

\subsection{Requerimientos prototipo ADS Libertad 2}

Como parte de la apropiación de conocimiento en el desarrollo de sistemas satelitales se hizo el ejercicio de seleccionar los sensores para la determinación de orientación del satélite Libertad 2, esto a pesar de que se va a usar el ADCS MAI-100 de la empresa Maryland Aerospace [25], el cual usa el magnetómetro PNI Micromag3 [26] y cuenta con entradas para el sensor de sol.

En general, se tiene que el satélite al ser liberado en el espacio desde el cohete de lanzamiento entrará en el modo Detumbling, momento en el cual el ADCS se enciende para estabilizar el satélite y así entrar en uno de sus modos de operación según la misión. El Libertad 2 tendrá una 
cámara como carga útil y un radio de comunicaciones que requiere apuntamiento a la estación terrera. Para lograr la correcta estabilización y orientación del satélite es necesario un cálculo preciso tanto en la determinación como en el control de la orientación.

Para este ADCS se propone el uso de sensores magnéticos y de giro, los cuales deben tener los siguientes requerimientos:

- Tamaño reducido

- Bajo consumo de energía

- Interfaz de comunicación digital SPI o I2C

- Temperatura de operación $-40{ }^{\circ} \mathrm{C} 85^{\circ} \mathrm{C}$

- 12 o más bits

- Magnetómetro de 3 ejes

- Giroscopio de 3 ejes

- Rango de medición del magnetómetro de mínimo $1 \mathrm{G}$ a máximo $2 \mathrm{G}$

- Rango de medición del giro de mínimo 1000 \% s a máximo 2000 \% s

- Sensibilidad del magnetómetro máximo en el orden de $\mathrm{mG}$

- Sensibilidad del giroscopios en el orden de máximo $1 \%$ s

- Variación lineal de la sensibilidad con respecto a la temperatura de máximo $5 \%$

\subsection{Selección de sensores prototipo ADCS Libertad 2}

$\mathrm{Al}$ realizar la búsqueda de magnetómetros en distribuidores de componentes electrónicos, se encontró que aquellos con tecnología MEMS eran adecuados por su pequeño tamaño, bajo consumo de energía y costo, por lo cual se pre-seleccionaron aquellos que usaran esta tecnología y que cumplieran con los requerimientos definidos previamente.

De los magnetómetros pre-seleccionados, se hicieron pruebas con el HMC5883L, el cual hace parte de la tarjeta de medida inercial Sparkfun 9 Degrees of Freedom - Sensor Stick (figura 1) y el MAG3110FCR1 de la tarjeta SEQUOIA SPACE Getting Started Board (figura 2).

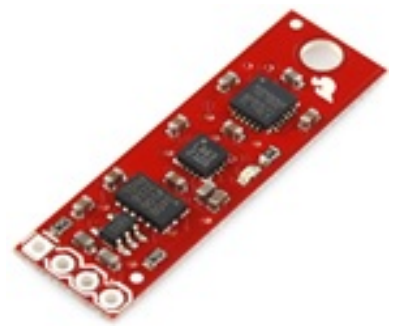

Figura 1: Unidad inercial Sparkfun 9 Degrees of Freedom Sensor Stick.

Teniendo en cuenta que el campo magnético terrestre no alcanza a llegar a 1 G y todos los magnetómetros consultados superan ese rango, la mejor opción sería usar el que menor rango de medición tenga dado que así tendrá una adecuada sensibilidad en la medición, llegando al orden de los $\mu \mathrm{G}$. Los sensores LSM303D y el LSM9DS0 se pueden configurar para trabajar a un rango de $2 \mathrm{G}$ con una sensibilidad de $80 \mu \mathrm{G} /$ bit, pero el LSM9DS0 es casi 3 veces más costoso por integrar en un solo chip sensor de aceleración en 3 ejes y sensor de giro en 3 ejes, mientras que el LSM303D solo integra un sensor de aceleración de 3 ejes.

En cuanto a los giroscopios, la tarjeta de Sparkfun usa el ITG-3200, mientras que el L3G4200D es usado en la tarjeta GSB de Sequoia. El L3G4200D pueden configurarse a diferentes rangos, por ende a diferentes sensibilidades y a la misma cantidad de bits, por lo cual los criterios de 




Figura 2: SEQUOIA SPACE Getting Started Board.

selección serán el precio y la integración con sensores magnéticos. La referencia LSM9DS0 que se mencionó previamente también es adecuada para el sensor de giro, por lo cual se elige este chip, el cual integra tres tipos de sensores en empaquetado pequeño, con bajo consumo de energía y de bajo precio. La integración de los sensores en un solo chip es adecuada para tener los ejes de referencia de cada uno de los sensores unidos a una sola referencia, algo que no se podría lograr al usar los sensores en chips separados.

Teniendo en cuenta las tarjetas que se tienen, se tienen 3 sensores de giro y 3 de campo magnético, a los cuales se les realiza pruebas de funcionamiento que involucran la configuración del sensor y la lectura de datos mediante comunicación I2C.

\subsection{Prueba de sensores seleccionados}

La prueba más sencilla que se puede realizar a cada uno de los sensores es configurándolos y leyendo datos, lo cual se hace mediante comunicación I2C. Las rutinas de configuración y lectura de datos son similares para cada uno de los sensores. En el caso de los magnetómetros se presenta variación en los datos de salida con tan solo acercar o alejar el sensor de fuentes magnéticas como computadores o celulares, o simplemente con acerca o alejar un imán. En cuanto a los sensores de giro los cambios se presentan con tan solo girar el sensor. Para ambos casos se probó la variación en las salidas para cada uno de los ejes de los sensores.

Una prueba más compleja se realizó al medir el campo magnético de un imán conforme se aleja del sensor. Para este caso se usó la tarjeta de Sparkfun, la GSB de Sequoia y un teslametro PHYWE 13610.90 como referencia para las medidas. El teslametro tiene una sensibilidad de $0.1 \mathrm{G}$ la cual es mucho menor a la que tienen los sensores de prueba, por lo cual representa un limitante en la toma de medidas.

Con el teslametro se midió un campo magnético de $62 \mathrm{mT}(620 \mathrm{G})$ en el imán, por lo cual para realizar las pruebas en los sensores se configuró un rango de $8 \mathrm{G}$ en la tarjeta Sparkfun y un rango de $10 \mathrm{G}$ en la tarjeta GSB de Sequoia. Se tomar medidas a unos $11 \mathrm{~cm}$ de distancia, lugar donde la intensidad del campo era menor a los $10 \mathrm{G}$ y a partir de ese punto se alejaba el imán $0.5 \mathrm{~cm}$ por lectura de muestras, que representan 10 datos por punto medido. Las mediciones se realizaron siempre midiendo la misma cara del imán y se repetía la operación al cambiar el eje de medición de los sensores.

Los resultados de las pruebas mostraron que para ambos sensores las curvas de datos medidas eran similares, mientras que la curva del teslametro se diferenciaba como se muestra en el ejemplo de la Figura 3

La diferencia que se presenta con las medidas realizadas por el Teslametro se pueden presentar par la baja sensibilidad que tiene, la cual es 50 veces peor que la del sensor de la tarjeta Sparkfun y 100 veces peor a la tarjeta GSB Sequoia. Es un claro ejemplo de las limitaciones que se presentan para realizar pruebas de ese tipo sin los recursos necesarios, por lo cual se recomienda seguir las especificaciones del fabricante. Con esta idea se recomienda usar el sensor LSM9DS0, por 


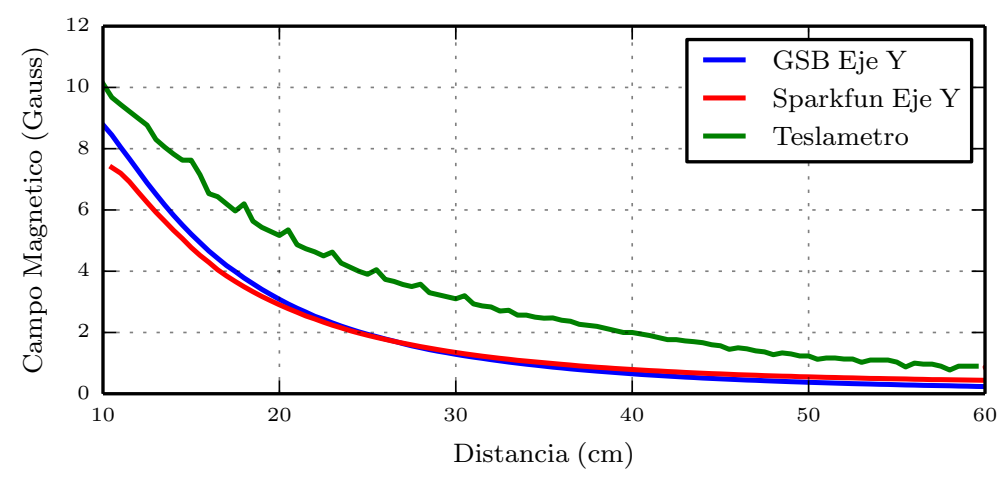

Figura 3: Campo magnético del imán medido en orientación al eje $Y$ de los sensores.

su pequeño tamaño. Si se compara el consumo de corriente de cada uno de los sensores (ver cuadro 1) sin contar el de los acelerómetros, se puede ver que son parecidos, por lo cual el sensor LSM9DS0 sigue siendo recomendado. Ante ese bajo consumo de corriente y la integración de 3 tipos de sensores en un solo chip de pequeño tamaño es un sensor adecuado para usar en un sistema de determinación de orientación como el que buscamos.

Tabla 1: Consumo de corriente de los sensores.

\begin{tabular}{|c|c|c|c|}
\hline Tarjeta & $I_{\text {Mag }}(\mu \mathrm{A})$ & $I_{\text {Giro }}(\mu \mathrm{A})$ & $I_{\text {Total }}(\mathrm{mA})$ \\
\hline Sparkfun & 100 & 6.5 & 6.6 \\
\hline GSB & 137.5 & 6.1 & 6.2 \\
\hline LSM9DS0 & 175 & 6.1 & 6.2 \\
\hline
\end{tabular}

\subsection{Consideraciones de diseño para el prototipo ADCS del Libertad 2}

La alineación de los ejes de referencia de los sensores con respecto a los ejes de referencia del satélite es importante, en la hoja de especificaciones de los sensores de 3 ejes se muestra la orientación de cada uno de sus ejes, teniendo en cuenta esto se deben alinear cada uno de los ejes del satélite con los ejes del sensor.

En cuanto al sensor de campo magnético, sus medidas se pueden ver afectadas por el campo magnético que generan los motores de las ruedas de reacción del sistema de control de orientación, razón por la cual se recomienda alejarlo lo más que se pueda de esa fuente de interferencia. En el caso del ADCS MAI-100, el manual de operación especifica que el magnetómetro debe ser colocado al menos a 5 pulgadas $(12.7 \mathrm{~cm})$ de distancia [25].

Ante la imposibilidad de adquirir un sensor de sol, la alternativa es el uso de fotodiodos o paneles solares. No se recomiendan fotorresistencias debido a su alta variación de voltaje con respecto a la temperatura, la cual cambia en amplio rango en el espacio. Si es necesario acondicionar alguna señal mediante amplificadores operacionales para llevarla a un voltaje determinado, verifique que los voltajes de alimentación del operacional se ajusten a su funcionamiento.

En el caso del MAI-100 se requiere que la salida de voltaje de los fotodiodos o de los paneles solares sea de $0 \mathrm{~V}$ para cuando no hay incidencia de luz y $5 \mathrm{~V}$ si hay incidencia directa de luz. Como prueba se usó el panel solar de la Figura 4 el cual es del tamaño de una unidad (1U) del estándar CubeSat y contiene 2 celdas solares conectadas en paralelo. La celda solar es de triple juntura con substrato de Arseniuro de Galio (GaAs) referencia 3G28C fabricado por la 
empresa alemana Azur Space [27], presentando una eficiencia $\eta$ del $28 \%$. Cada una de las celdas genera $2.37 \mathrm{~V}$ con radiación directa del sol a máxima potencia de energía entregada por la celda, según mediciones realizadas en la tesis de grado del ingeniero electrónico Sergio Andrés Sánchez Sanjuán [28], por lo cual se puede acondicionar la señal para que esté en el rango de $0 \mathrm{~V}-5 \mathrm{~V}$ mediante el uso de un amplificador operacional en configuración no inversora.

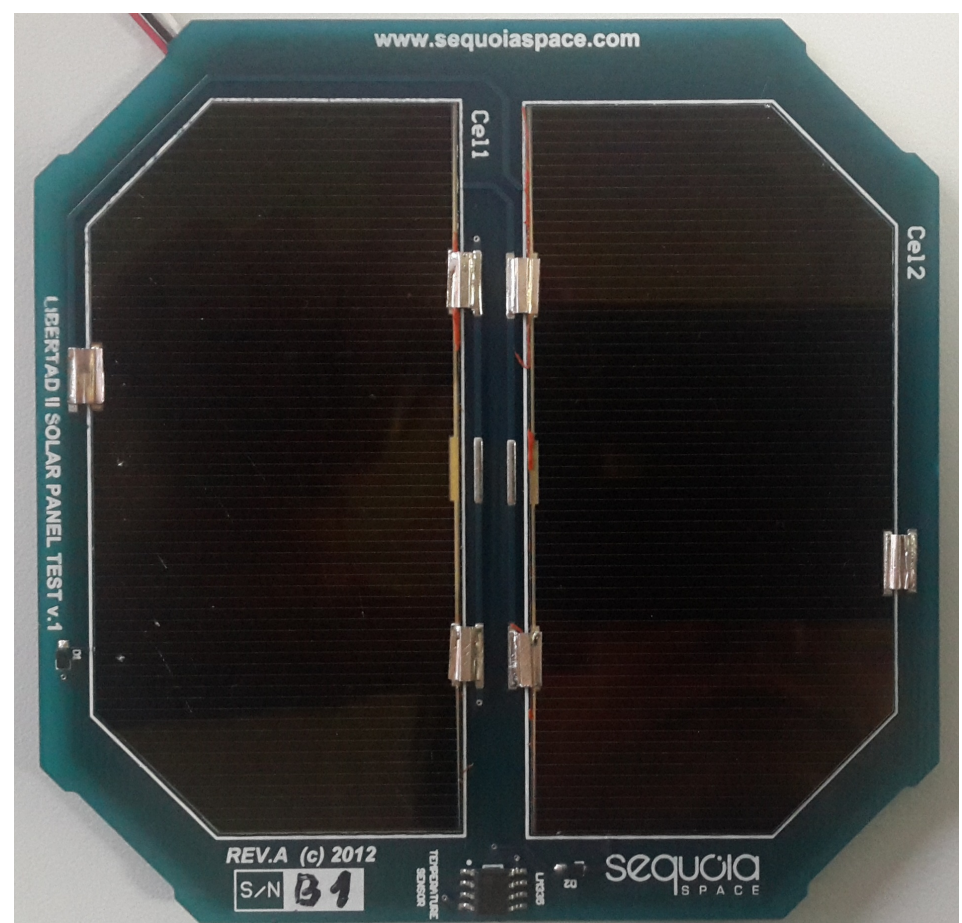

Figura 4: Panel solar 1 unidad estándar CubeSat.

Al usar una configuración de amplificador no inversor es posible encontrar un operacional que puede alimentar con fuente sencilla, sin necesidad de usar voltaje negativo, solamente se requiere de voltaje positivo y tierra. La alimentación de voltaje puede variar dependiendo del nivel máximo de voltaje que se quiere de salida, en este caso se requiere una máxima de $5 \mathrm{~V}$, por lo cual para poder realizar la amplificación a ese nivel de voltaje la alimentación debe ser superior a los $5 \mathrm{~V}$ para evitar la saturación del operacional, debido a esto se considera usar el voltaje de $12 \mathrm{~V}$, que también el alimenta el MAI-100.

La Figura 5 muestra el diseño de la configuración no inversora. El voltaje de la fuente V1 es de $+12 \mathrm{~V}$, mientras que el voltaje de la fuente $\mathrm{V} 3$ es $0 \mathrm{~V}$, por lo tanto se puede considerar como tierra (dado que no se necesita una fuente de voltaje negativo). La fuente de voltaje V2 es el voltaje generado por el fotodiodo o por la celda solar, e ingresa por la entrada positiva del operacional.

En el circuito hay 3 resistencias $\left(R_{1}, R_{2}\right.$ y $\left.R_{3}\right)$, las resistencias $R_{1}$ y $R_{2}$ definen la ganancia $(G)$ de la señal de entrada $\left(V_{\text {in }}\right)$. La resistencia $R_{1}$ realimenta el voltaje de salida $\left(V_{\text {out }}\right)$ a la entrada inversora del operacional. La ganancia $(G)$ de la señal de salida $\left(V_{\text {out }}\right)$ se da por la ecuación 1:

$$
G=\frac{V_{\text {out }}}{V_{\text {in }}}=1+\frac{R_{1}}{R_{2}}
$$

Para el caso de las celdas solares que entregan un voltaje máximo cercano a $2.5 \mathrm{~V}$ a máxima potencia, se requiere una ganancia de $2(G=2)$ para llegar a los $5 \mathrm{~V}$. Con lo que se obtiene que: $R_{2}=R_{1}$. 


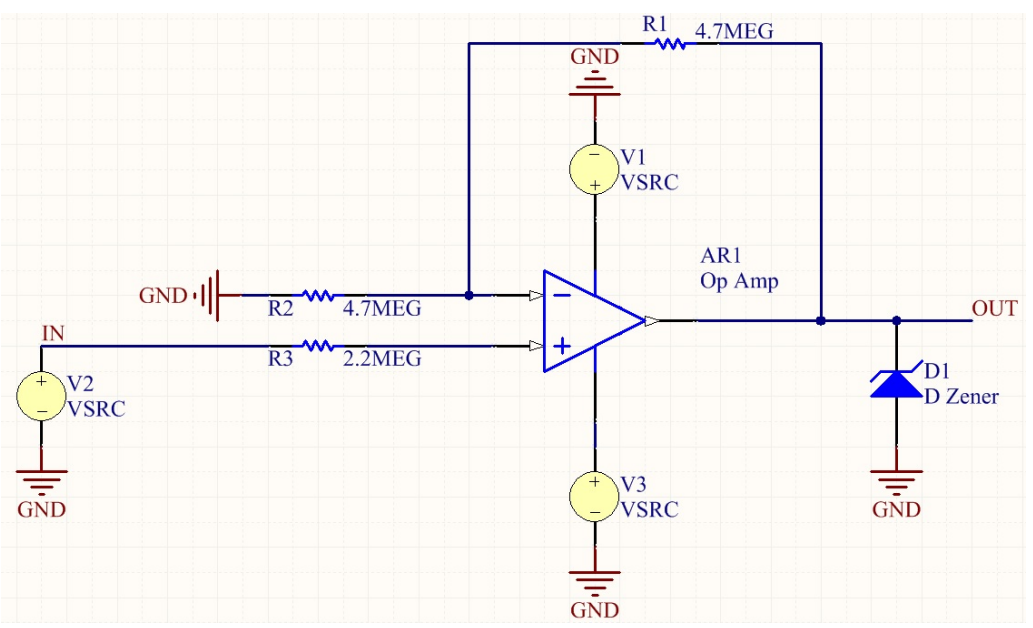

Figura 5: Diseño circuito amplificador operacional no inversor para el acondicionamiento de la señal del fotodiodo.

Para hallar el valor de la resistencia $R_{3}$ se usa la ecuación 2:

$$
R_{3}=\frac{R_{1} R_{2}}{R_{1}+R_{2}}
$$

Para evitar que este circuito consuma mucha corriente, la cual es limitada en el satélite, se recomienda que las resistencias sean de muy alto valor para que la impedancia de entrada sea alta y así la corriente de entrada sea muy baja.

Para probar el rango de voltaje generado por la celda solar junto con el circuito de amplificación se midió el voltaje de salida en ausencia de luz. Se tomaron valores de voltaje de salida variando el ángulo de incidencia de la luz generada por una fuente artificial a una distancia de $5 \mathrm{~cm}$ de la celda solar, con el objetivo de ver la variación de voltaje con respecto al ángulo de incidencia de la luz.

Hay que tener en cuenta que las mediciones se realizan usando la celda solar sin ningún tipo de carga, por lo cual según las especificaciones del fabricante, puede generar un voltaje máximo de $5 \mathrm{~V}$. Cabe aclarar que a máxima potencia la celda puede generar $2.37 \mathrm{~V}$ cuando está entregando energía al satélite, por lo cual la configuración de ganancia del operacional se debe ajustar a ese voltaje. Para nuestro caso de prueba asumimos que el máximo voltaje entregado a máxima potencia es de $2.5 \mathrm{~V}$ por lo cual se ajusta el operacional a una ganancia de $G=2$.

Teniendo en cuenta lo anterior, en ausencia de luz el voltaje de salida es de $0 \mathrm{~V}$, y en luz ambiente el voltaje de salida es aproximadamente de $4 \mathrm{~V}$. Al exponer la celda a luz del sol con cielo nublado se obtiene un voltaje aproximado de $8 \mathrm{~V}$. Con respecto a las mediciones de voltaje tomadas para la incidencia de luz con la fuente artificial a determinados ángulo se obtienen los resultados presentes en la Figura 6.

Para el eje horizontal se tiene que los cero grados $\left(0^{\circ}\right)$ es cuando la luz está al lado derecho de la celda y va en paralelo a ella, por lo cual el valor del voltaje de salida debe ser el generado por la luz ambiente del laboratorio, que para nuestro caso es de aproximadamente $4 \mathrm{~V}$. Cuando el ángulo de incidencia es de $90^{\circ}$ la luz de la fuente tiene una incidencia normal sobre la celda solar y el voltaje de salida se aproxima a los $5 \mathrm{~V}$. Por último cuando el ángulo de incidencia es de $180^{\circ}$ la luz de la fuente no incide sobre la celda, por lo tanto el voltaje de salida es el de la luz ambiente. De la gráfica se puede ver que el voltaje entregado por la celda varía con respecto al ángulo de incidencia de la luz, donde su máximo voltaje se entrega cuando la radiación es 


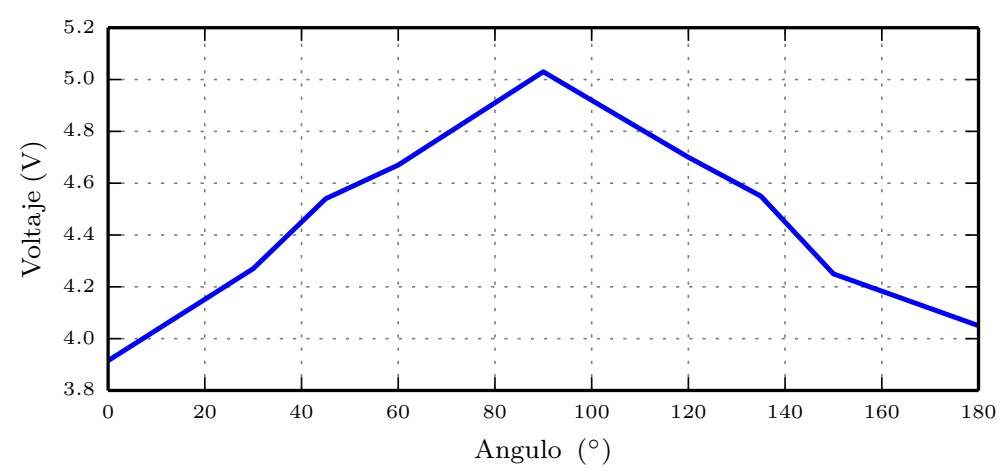

Figura 6: Comportamiento del voltaje de salida de la cerda solar respecto al ángulo de incidencia de la luz.

directa a la celda y decrece con respecto al ángulo. Lo que muestra que las celdas se pueden usar como sensor de Sol en un satélite como el nuestro.

Por último se propone el uso de un diodo Zener (D1 en la figura 5) de $5 \mathrm{~V}$ para limitar el voltaje de salida en caso de que se presente problemas con el voltaje de entrada.

\section{Conclusiones y trabajos futuros}

La experiencia alcanzada en la selección de sensores para un prototipo de sistema de determinación de orientación para pequeños satélites es presentada en este artículo. Se contempla la definición y análisis de requerimientos del sistema de determinación de orientación para un CubeSat de $3 \mathrm{U}$, la selección y prueba de los sensores, y algunas consideraciones a tener en cuenta en el diseño de este sistema.

Los bajos costos que representa el desarrollo de un satélite tipo CubeSat, ha permitido que instituciones académicas puedan participar en este tipo de proyectos. Sin embargo la información que guía el desarrollo de misiones satelitales se enfoca en proyectos de gran envergadura, los cuales se ajustan de manera limitada al desarrollo de un satélite pequeño como un CubeSat.

Diversas instituciones académicas que han desarrollado sistemas de determinación de orientación, presentan las especificaciones de los desarrollos que han hecho y de los resultados que han obtenido, pero no la forma cómo los desarrollaron, lo cual limita el aporte en conocimiento que puede recibir alguien interesado en participar en este sistema y sin tener experiencia.

\section{Referencias}

[1] L. Martin, W. H. Jones, and W. A. Shiroma, "Small-Satellite Projects Offer Big Rewards," Potentials, IEEE, vol. 33, no. 4, pp. 24-30, 2014.

[2] J. Puig-Suari, C. Turner, and W. Ahlgren, "Development of the standard CubeSat deployer and a CubeSat class $\backslash$ nPicoSatellite," 2001 IEEE Aerospace Conference Proceedings (Cat. No.01TH8542), vol. 1, pp. 347-353, 2001.

[3] J. Puig-Suari, C. Turner, and R. J. Twiggs, "CubeSat: The Development and Launch Support Infrastructure for Eighteen Different Satellite Customers on One Launch," in 15th Annual/USU Conference on Small Satellites, pp. 1-5, 2001.

[4] The CubeSat Program, "CubeSat Design Specification Rev. 13," 2014.

[5] J. Bouwmeester and J. Guo, "Survey of worldwide pico- and nanosatellite missions, distributions and subsystem technology," Acta Astronautica, vol. 67, no. 7-8, pp. 854-862, 2010.

[6] H. Heidt, J. Puig-Suari, A. S. Moore, P. S. Nakasuka, and R. J. Twiggs, "CubeSat: A New Generation of Picosatellite for Education and Industry Low-Cost Space Experimentation," in 14th Annual/USU Conference on Small Satellites, (Logan, USA), pp. 1-19, 2000.

[7] K. Woellert, P. Ehrenfreund, A. J. Ricco, and H. Hertzfeld, "Cubesats: Cost-effective science and technology 
platforms for emerging and developing nations," Advances in Space Research, vol. 47, no. 4, pp. 663-684, 2011.

[8] X. Yu, J. Zhou, P. Zhu, and J. Guo, "Star of AOXiang: An innovative 12U CubeSat to demonstrate polarized light navigation and microgravity measurement," Acta Astronautica, vol. 147, no. March, pp. 97-106, 2018.

[9] NASA/Goddard Space Flight Center, "Firefly: An NSF CubeSat project. Understanding Earth's most powerful natural particle accelerator," 2018.

[10] J. G. F. D. A. G. J. Soliz Torrico, R. Hurtado and Y. Villanueva, "Libertad 2; colombian satellite for earth observation," in International Astronautical Congress, 2014. Proceedings., 2014 IAC, vol. 1, pp. 3355-3359 vol.1, Sep 2014.

[11] J. R. Wertz, Spacecraft Attitude Determination and Control. D. Reidel, 1 ed., 1980.

[12] M. J. Sidi, Spacecraft Dynamics \& Control. Cambridge, 1997.

[13] Universidad Sergio Arboleda, "Proyecto Espacial Colombia en Órbita, Primer Satélite Colombiano Libertad $1, " 2007$.

[14] M. Cheguini and F. Ruiz, "Real-time attitude estimation based on Gradient Descent algorithm," in Circuits and Systems (CWCAS), 2012 IEEE 4th Colombian Workshop on, pp. 1-6, nov 2012.

[15] A. Slavinskis, E. Kulu, J. Viru, R. Valner, H. Ehrpais, T. Uiboupin, M. Jarve, E. Soolo, J. Envall, T. Scheffle, I. Sunter, H. Kuuste, U. Kvell, J. Kalde, K. Laizans, E. Ilbis, T. Eenmae, R. Vendt, K. Voormansik, I. Ansko, V. Allik, S. Latt, and M. Noorma, "Attitude determination and control for centrifugal tether deployment on the ESTCube-1 nanosatellite," 2014.

[16] S. Rossi, A. Ivanov, G. Burri, V. Gass, C. Hol, and M. Rothacher, "CUBETH SENSOR CHARAC TERIZATION : SENSOR A NALYSIS REQUIRED FOR A CUBES AT MISSION," in 2nd IAA Conference on Dynamics and Control of Space Systems, (Rome, Italy), pp. 1-20, 2014.

[17] P. J. Tassano Matias , Monzon Pablo, "ATTITUde DETERMinATION AND CONTROL SYSTEM OF THE URUGUAYAN CUBESAT, ANTELSAT," Advanced Robotics (ICAR), 2013 16th International Conference on, vol. 01, 2013.

[18] G. F. De Oliveira, J. Y. Ishihara, R. a. Borges, H. C. Ferreira, A. M. Kulabukhov, V. a. Larin, and V. V. Belikov, "A Low-Cost Attitude Determination and Control System for the UYS-1 nanosatellite," IEEE Aerospace Conference Proceedings, 2013.

[19] J. C. Springmann, A. J. Sloboda, A. T. Klesh, M. W. Bennett, and J. W. Cutler, "The attitude determination system of the RAX satellite," Acta Astronautica, vol. 75, pp. 120-135, jun 2012.

[20] S. Ghuffar, Design and Implementation of Attitude Determination Algorithm for the Cubesat UWE-3. PhD thesis, University of Wuerzburg Faculty, 2010.

[21] J. G.-L. F. Sierra-Sánchez, S. Pérez-Munevar, R. Hurtado-Velasco, "Guía Metodológica para el Diseño de Subsistemas de Potencia y Orientación en NanoSatélites," Revista De Ciencia E Ingeniería Del Instituto Tecnológico Superior De Coatzacoalcos, vol. 2, pp. 86 - 91, 2015.

[22] J. Wertz, D. Everett, and J. Puschell, eds., Space Mission Engineering: The New SMAD. Space technology library, Microcosm Press, 2011.

[23] W. Larson and J. Wertz, Space Mission Analysis and Design. Microcosm Press and Kluwer Academic Publishers, third ed., 2005.

[24] NASA, "Outgassing Data for Selecting Spacecraft Materials Online," 2018.

[25] Maryland Aerospace Inc., MAI-100 MiniADACS System Operations Manual. No. 410, 2012.

[26] PNI Corporation, "Micromag3 Data Sheet," 2005.

[27] Azur Space, "Azur Space Solar Power GMBH, 28\% Triple Junction GaAs Solar Cell specications," 2009.

[28] S. A. Sánchez Sanjuán, "Modelamiento y simulación de la energía de un satélite CubeSat tipo 3U en diferentes escenarios de orientación para una órbita terrestre baja," 2014.
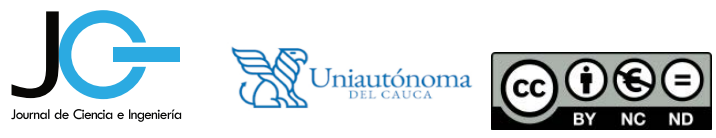